\title{
Modulation of Abnormal Metabolic Brain Networks by Experimental Therapies in a Nonhuman Primate Model of Parkinson Disease: An Application to Human Retinal Pigment Epithelial Cell Implantation
}

\author{
Shichun Peng*1, Yilong Ma*1, Joseph Flores ${ }^{2}$, Michael Cornfeldt ${ }^{3}$, Branka Mitrovic ${ }^{4}$, David Eidelberg ${ }^{\dagger 1}$, \\ and Doris J. Doudet ${ }^{\dagger 2}$ \\ ${ }^{1}$ Center for Neurosciences, The Feinstein Institute for Medical Research, Manhasset, New York, New York; ${ }^{2}$ Department of Neurology, \\ University of British Columbia, Vancouver, British Columbia, Canada; ${ }^{3}$ Titan Pharmaceuticals Inc., Somerville, New Jersey; and \\ ${ }^{4}$ Bayer HealthCare Pharmaceuticals Inc., Richmond, California
}

Abnormal covariance pattern of regional metabolism associated with Parkinson disease (PD) is modulated by dopaminergic pharmacotherapy. Using high-resolution ${ }^{18} \mathrm{~F}-\mathrm{FDG}$ PET and network analysis, we previously derived and validated a parkinsonism-related metabolic pattern (PRP) in nonhuman primate models of PD. It is currently not known whether this network is modulated by experimental therapeutics. In this study, we examined changes in network activity by striatal implantation of human levodopa-producing retinal pigment epithelial (hRPE) cells in parkinsonian macaques and evaluated the reproducibility of network activity in a small test-retest study. Methods: ${ }^{18} \mathrm{~F}-\mathrm{FDG}$ PET scans were acquired in 8 healthy macaques and 8 macaques with 1-methyl-4-phenyl-1,2,3,6-tetrahydropyridine (MPTP)-induced bilateral nigrostriatal dopaminergic lesions after unilateral putaminal implantation of hRPE cells or sham surgery. PRP activity was measured prospectively in all animals and in a subset of test-retest animals using a network quantification approach. Network activity and regional metabolic values were compared on a hemispheric basis between animal groups and treatment conditions. Results: All individual macaques showed clinical improvement after hRPE cell implantation compared with the sham surgery. PRP activity was elevated in the untreated MPTP hemispheres relative to those of the normal controls $(P<$ $0.00005)$ but was reduced $(P<0.05)$ in the hRPE-implanted hemispheres. The modulation observed in network activity was supported by concurrent local and remote changes in regional glucose metabolism. PRP activity remained unchanged in the untreated MPTP hemispheres versus the sham-operated hemispheres. PRP activity was also stable $(P \geq 0.29)$ and correlated $\left(R^{2} \geq 0.926 ; P<0.00005\right)$ in the test-retest hemispheres. These findings were highly reproducible across several PRP topographies generated in multiple cohorts of parkinsonian and healthy macaques. Conclusion: We have demonstrated long-term therapeutic effects of hRPE cell implantation in nonhuman primate models of PD. The implantation of such levodopa-producing cells can concurrently decrease the elevated metabolic network activity in parkinsonian brains on an individual basis. These results parallel the analogous findings

Received Jul. 7, 2015; revision accepted Mar. 7, 2016.

For correspondence or reprints contact: Yilong Ma, The Feinstein Institute for Medical Research, 350 Community Dr., Manhasset, NY 11030.

E-mail: yma@nshs.edu

${ }^{*}$ Contributed equally to this work.

tContributed equally to this work.

Published online Apr. 7, 2016.

COPYRIGHT (C) 2016 by the Society of Nuclear Medicine and Molecular Imaging, Inc. reported in patients with PD undergoing levodopa therapy and other symptomatic interventions. With further validation in large samples, ${ }^{18} \mathrm{~F}-\mathrm{FDG}$ PET imaging with network analysis may provide a viable biomarker for assessing treatment response in animal models of PD after experimental therapies.

Key Words: parkinsonism; primate model; FDG PET; network analysis; dopamine cell transplantation; retinal pigment epithelial cell

J Nucl Med 2016; 57:1591-1598

DOI: 10.2967/jnumed.115.161513

$\mathbf{T}$ he motor clinical manifestations of Parkinson disease (PD) are attributed chiefly to progressive loss of nigrostriatal dopaminergic neurons. This degenerative process causes a deficiency of endogenous dopamine, leading to impairment in the cortico-striatothalamo-cortical motor circuitry. ${ }^{18}$ F-FDG PET has been widely used to study functional abnormality in this circuitry. Motor features of PD are associated with an abnormal metabolic brain network (i.e., Parkinson disease-related pattern [PDRP]) characterized by increased activity in pallidothalamic, pontocerebellar, and motor cortical regions and decreased activity in the posterior parietooccipital cortices (1-4). The network activity measured prospectively in individual patients is increased relative to controls and correlates positively with the severity of motor symptoms. This network is also modulated by dopaminergic and neurosurgical treatments in PD patients (5-7) predicting the clinical outcome.

The nonhuman primate injected with 1-methyl-4-phenyl-1,2,3,6tetrahydropyridine (MPTP) is the most common primate model of experimental parkinsonism. ${ }^{18} \mathrm{~F}$-FDG PET is useful for quantifying specific subcortical and cortical metabolic changes in MPTP primate models as a result of neurotoxic lesions or therapeutics $(8,9)$. We derived and validated a parkinsonism-related pattern (PRP) in multiple cohorts of parkinsonian and normal monkeys (10). PRP was analogous to PDRP in patients in terms of topographic features, group discrimination, and clinical correlation $(5,7)$. It is currently not known whether this network is modulated by experimental therapies in the MPTP primate model of PD.

Human retinal pigment epithelial (hRPE) cells of fetal origin, attached to gelatin microcarriers (GM; hrPE-GM) for enhanced 
survival, have been used as a potential therapy to reverse parkinsonian motor deficits in rodents $(11,12)$, in monkeys $(13,14)$, and, under the name Spheramine (Titan Pharmaceuticals, Inc.), in PD patients $(15,16)$. There is unequivocal evidence that hRPE-GM implants can survive without immunosuppression $(17,18)$ as in fetal cell transplantation in PD patients (19). Increased ${ }^{18} \mathrm{~F}$-fluorodopa uptake and decreased ${ }^{11} \mathrm{C}$-raclopride binding were reported in the implanted striatum after unilateral implant of hRPE-GM in monkeys, suggesting a dopaminergic mechanism (14). Despite sustained improvement in clinical motor scores over several years, these molecular markers are highly variable over the long term because of inherent compensatory modulation after therapy and disease progression in patients $(20,21) .{ }^{18} \mathrm{~F}-\mathrm{FDG}$ PET imaging may be more sensitive for examining systemic brain function recovery after dopaminergic cell implantation. However, there are presently no studies on the metabolic effects of dopaminergic implants in parkinsonian animals or patients.

In this study, we describe a general approach to assessing network and regionally specific metabolic responses to experimental therapies in nonhuman primate models of parkinsonism. We hypothesized that metabolic network is modulated by striatal cell implantation via widespread downstream changes in brain circuitry associated with motor function recovery. To this end, we evaluated the effects of hRPE-GM implants on metabolic brain network activity and regional metabolism in parkinsonian macaques. The test-retest stability of network activity was also assessed prospectively in a small cohort.

\section{MATERIALS AND METHODS}

\section{Characteristics of Macaques}

This study included 16 adult rhesus monkeys (males, 14; females, 2; age, 8-22 y). Eight monkeys (mean age $\pm \mathrm{SD}, 13.5 \pm 5.2 \mathrm{y}$; mean weight $\pm \mathrm{SD}, 9.1 \pm 1.7 \mathrm{~kg}$ ) developed mild to moderately severe bilateral parkinsonism after chronic intravenous administration of MPTP over several months. hRPE-GM or sham (GM only) was implanted unilaterally in the striatum in the fully recovered and stable animals (i.e., motor scores unchanged for $>3-4$ mo). Controls comprised 8 healthy monkeys (age, $10.8 \pm 4.7 \mathrm{y}$; weight, $9.7 \pm 2.7 \mathrm{~kg}$ ) matched in age $(P=0.28)$ and weight $(P=0.63)$ to the parkinsonian animals. Clinical evaluation and ${ }^{18}$ F-FDG PET were performed in all animals and repeated in a small cohort of 4 subgroups (Table 1; Supplemental Tables 1 and 2 [supplemental materials are available at http:// jnm.snmjournals.org]). Part of these imaging data was used previously for the determination and validation of PRP (10). All the procedures followed the Canadian Council on Animal Care Regulations and were approved by the Committee on Animal Care of the University of British Columbia.

The parkinsonian and healthy monkeys lived in groups or pairs and were able to forage and care for themselves. Motor scores of these animals were obtained using a reliable clinical rating scale (14), equivalent to the unified Parkinson disease rating scale with a maximal score of 26. All MPTP-lesioned animals presented bilateral parkinsonian symptoms with generally reduced activity, hypokinesia, bradykinesia, tremor, and deficits in balance and coordination. The parkinsonian animals were evaluated before and 6-48 mo after unilateral striatal implantation (Fig. 1A) and rated as mild moderate $(n=7)$ or moderately severe $(n=1)$ at the time of imaging (motor score, $11.9 \pm 5.1$; range, 8-23).

Assessment of glucose metabolic networks was not part of the initial hRPE study of unilateral implantation efficacy, and the animals were only scanned late in the clinical study as a proof of concept. Network activities were thus compared between implanted and nonimplanted hemispheres as well as with healthy controls.

\section{Protocols of hRPE Cell Preparation and Implantation}

The preparation of hRPE-GM and GM slurry and sterile surgical and implantation procedures were described elsewhere $(14,17)$. This study used hRPE cells from 2 different sources (Table 1). The initial cells provided by Titan Pharmaceuticals came from a fetal donor of an age (22-24 wk) similar to those used in the small open-label clinical phase I trial (16). Later cells supplied by Bayer Schering Pharma came from 2 different neonate donors (32-34 wk) similar in age and preparation to those used in the large double-blind clinical phase II trial (22).

The nonimmunosuppressed parkinsonian monkeys received unilateral injections of hRPE-GM $(n=6)$ or GM alone $(n=3)$ randomly in the left or right striatum. The implantation of GM was performed only in a small number of animals to confirm the finding in the original safety and efficacy study (13). One animal was first implanted with GM in one striatum and after several months without notable clinical improvement, received hRPE-GM in the contralateral striatum. The animals were sedated with ketamine $(10 \mathrm{mg} / \mathrm{kg}$ intramuscularly) for

TABLE 1

Parkinsonian Macaque Characteristics and Imaging-Related Parameters

\begin{tabular}{|c|c|c|c|c|c|c|c|}
\hline ID & Age (y) & Weight (kg) & Motor rating & Dose (MBq) & Glucose (mmol/L) & Condition, left & Condition, right \\
\hline 1 & 22 & 9 & 10 & 281 & 4.1 & $\mathrm{hRPE}^{\star}$ & MPTP \\
\hline 2 & 13 & 8 & 8 & 148 & 3.7 & $\mathrm{hRPE}^{\star}$ & MPTP \\
\hline 3 & 13 & 10 & 8 & 185 & 3.3 & $\mathrm{hRPE}^{\star}$ & MPTP \\
\hline 4 & 20 & 10 & 14 & 211 & 3.7 & $\mathrm{hRPE}^{\star}$ & MPTP \\
\hline 5 & 9 & 12 & 10 & 244 & 3.6 & MPTP & $\mathrm{hRPE}^{\dagger}$ \\
\hline 6 & 14 & 7 & 8 & 185 & 4.1 & $\mathrm{hRPE}^{\dagger}$ & GM \\
\hline 7 & 8 & 7 & 23 & 185 & 3.5 & GM & MPTP \\
\hline 8 & 9 & 10 & 14 & 222 & 4.5 & MPTP & GM \\
\hline
\end{tabular}

*Fetal cells.

${ }^{\dagger}$ Neonatal cells.

Data are provided for macaques undergoing ${ }^{18} \mathrm{~F}-\mathrm{FDG}$ PET after unilateral striatal implantation of hRPE cells or gelatin carriers only (GM) in left or right striatum. 

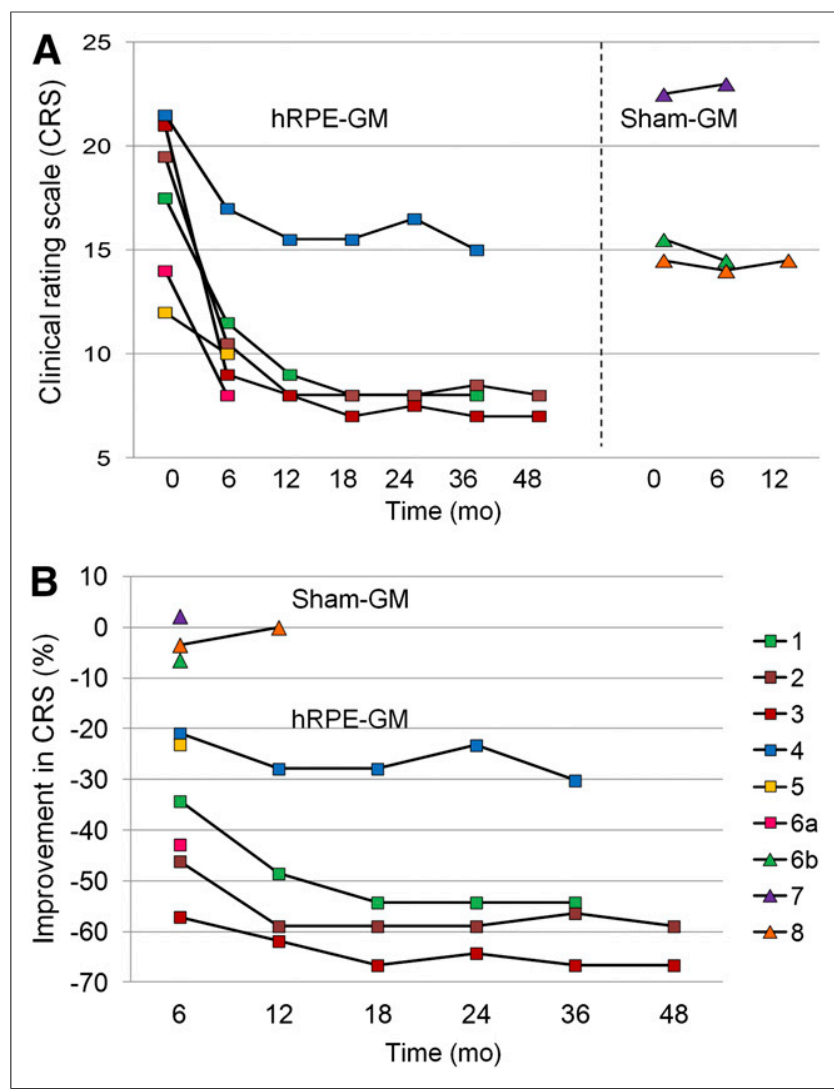

FIGURE 1. Clinical improvement in individual macaques after cell-based therapy. hRPE-implanted animals showed motor recovery from 6 mo to $4 \mathrm{y}$ after unilateral implantation but continued to express mild to moderate bradykinesia and hypokinesia. Maximal benefit was achieved within $1 \mathrm{y}$ and remained stable afterward. Sham-implanted animals showed no clinical responses. Animal 6 was transplanted sequentially with $\mathrm{GM}$ and hRPE-GM in 2 different hemispheres. Animals 1-4 received fetal cells used in the successful phase I trial (15), and animals 5-6a received neonatal cells used in the failed phase II trial (22). - = hRPE-GM; $\boldsymbol{\Delta}=\mathrm{GM}$.

placement of intravenous lines and intubation and anesthetized (isoflurane, $2 \%-3 \%$ ). The animals received prophylactic antibiotics and analgesics before and after the procedure. Each animal was placed in a MRI-compatible stereotactic frame with the implant coordinates determined from an MR image obtained immediately before the surgery. Each animal received 5 tracks, $2 \mathrm{~mm}$ apart, in a zig-zag pattern covering the extent of the putamen or posterior caudate/putamen. Fifty microliters of slurry with $65,000-100,000 \mathrm{hRPE}$ cells attached to GM or GM alone were injected in each track. No adverse events were observed in any of the animals after hRPE-GM or sham implantation.

\section{PET Imaging}

PET imaging was performed using a high-resolution research tomograph (HRRT; CPS Innovations) with a 3-dimensional resolution of $2.5 \mathrm{~mm}(10)$. The monkey and his/her partner were moved to smaller squeeze cages in an adjacent room to facilitate radiotracer administration. ${ }^{18}$ F-FDG (148-222 MBq) was injected intramuscularly in the left or right thigh. The animals stayed awake during an uptake period of 40-45 min, and none exhibited abnormal behavior or marked motor activity in the videorecording. The monkey was then rapidly anesthetized (ketamine followed by isoflurane for maintenance). A 30-min emission scan followed by a 10-min attenuation scan were acquired from $80 \mathrm{~min}$ after injection and reconstructed using the ordered-subsets expectation maximization algorithm. There were no differences in injected dose $(211.4 \pm 22.7$ vs. $207.7 \pm 41.5$ MBq; $P=0.83)$ and blood glucose $(3.96 \pm 0.52$ vs. $3.81 \pm 0.39$ $\mathrm{mmol} / \mathrm{L} ; P=0.52$ ) values between the healthy and all parkinsonian animals (Table 1; Supplemental Table 1).

\section{Image Processing}

Image processing was performed with in-house program (ScanVP available at http://www.feinsteinneuroscience.org) and statistical parametric mapping software (SPM; Institute of Neurology). PET images were spatially normalized into a macaque brain template (23) and smoothed with a 4-mm gaussian filter. To assess the metabolic effects of unilateral implants, these images were separated into hemispheres and flipped onto the same side. The left and right hemispheric images were averaged for the animals that were healthy or had only bilateral MPTP lesions, making the number of hemispheres in the analysis equal to that of animals (Fig. 2).

\section{Reproducible PRP Metabolic Brain Networks Across Animal Cohorts}

We previously established several metabolic patterns (PRP1 to PRP5) in 2 independent cohorts of parkinsonian and healthy rhesus macaques (10). PRPs 1-4 were generated over hemispheres. The hemispheric PRPs (i.e., PRP1) were topographically comparable to symmetric PRP5 produced over whole brains in the parkinsonian and control animals available. These patterns corresponded to the MPTP animals with moderate to severe (PRP1, PRP2, PRP5) and mild to moderate (PRP3, PRP4) motor symptoms. All PRPs shared a similar topography characterized by positive activity in striatal, pallidothalamic, pons, and motor cortical regions and negative activity in the posterior parietal-occipital cortex (Fig. 3A; Supplemental Fig. 1A). Network activity in individual hemispheres or brains discriminated

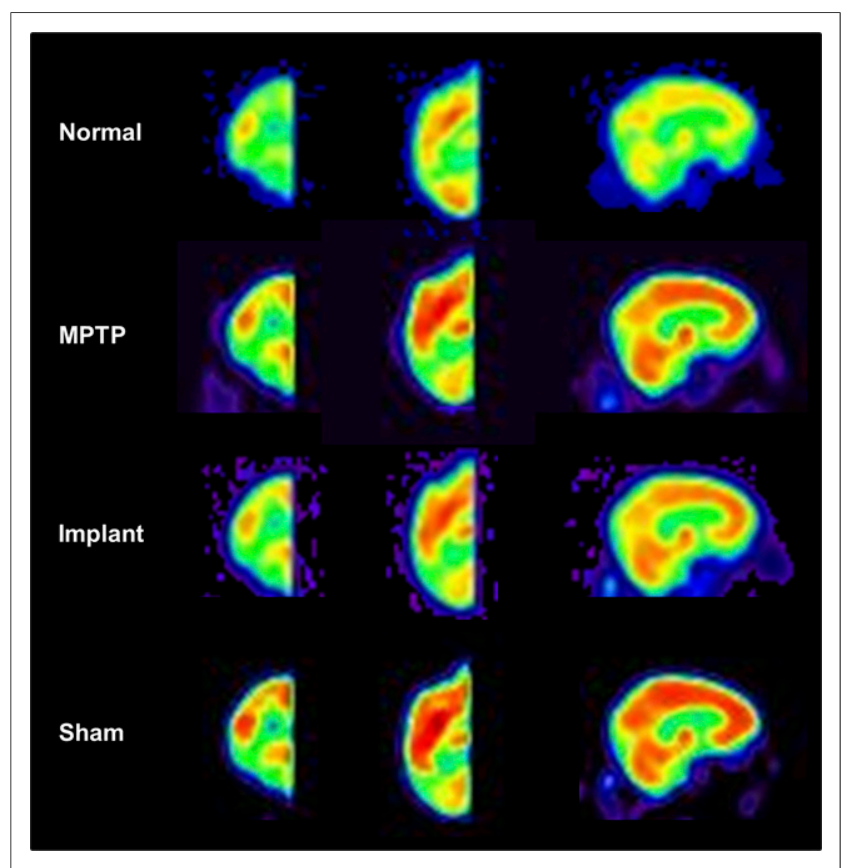

FIGURE 2. Mean images of relative glucose metabolism in healthy and parkinsonian macaques acquired using Siemens HRRT scanner. This high-resolution PET scanner provides superior image quality for revealing distinct regional differences in cortical and subcortical metabolism among normal, MPTP, and hRPE- and sham-implanted hemispheres. Each image represents brain ${ }^{18} \mathrm{~F}-\mathrm{FDG}$ scans averaged over hemispheres in individual animal group spatially normalized to macaque PET brain template (23). 


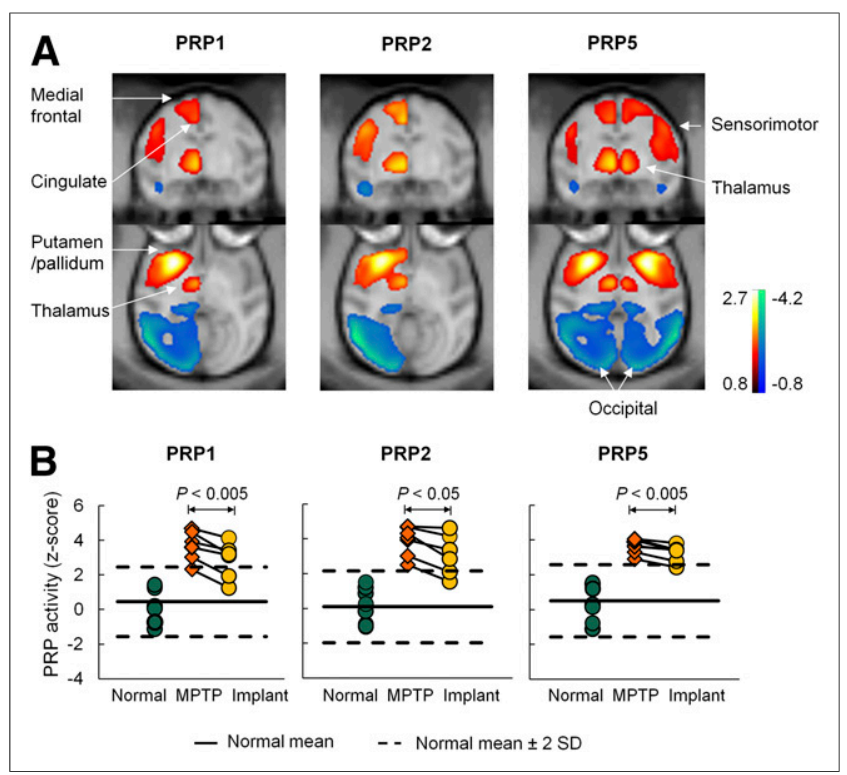

FIGURE 3. Modulation of abnormal metabolic brain networks in MPTP-induced experimental parkinsonism by hRPE cell transplantation therapy. (A) PRPs identified on a hemispheric (PRPs 1 and 2) and wholebrain (PRP 5) basis using ${ }^{18} \mathrm{~F}$-FDG PET images in parkinsonian and agematched healthy macaques (10). All PRPs shared analogous topographies with increased (red to yellow) and decreased (blue to green) metabolic activity in subcortical and cortical regions. (B) Network activity in individual hemispheres or brains was elevated $(P<0.00005)$ in the 7 untreated MPTP hemispheres compared with the 8 normal controls but declined consistently $(P<0.05)$ in the 6 contralateral MPTP hemispheres after hRPE cell implantation. The patterns are overlaid on macaque MRI brain template (23).

MPTP and normal animals in the derivation sample $(P<0.005)$ for each PRP (Table 2; Supplemental Fig. 3).

\section{Changes in PRP Network Activity in Treatment Sample}

The treatment sample included the 8 MPTP monkeys unilaterally treated with hRPE-GM or GM implantation. We quantified the expression of each PRP (i.e., PRPs 1-5) prospectively in individual hemispheres masked to animal, clinical symptom, and treatment status $(1,4)$. The network score of PRP5 was computed in the whole brains but over hemispheres in the images involving unilateral interventions such as MPTP lesion, hRPE, or sham implants. All network scores were z-transformed using analogous PRP scores for the healthy animals in the derivation cohort so that they had a mean of 0 and SD of 1. Network scores were compared among 8 normal, 7 MPTP, and 6 hRPE-implanted hemispheres.

\section{Changes in PRP Network Activity in Test-Retest Sample}

The test-retest sample included a mixed group of 7 normal, MPTP, implant, and sham animals imaged twice over $0.94 \pm 0.73$ y (Supplemental Table 2). Animal weight, injected dose, and blood glucose level did not change between the 2 time points $(P \geq 0.17)$; neither did motor scores in the parkinsonian animals $(P>0.11)$. Network scores of all PRPs were computed in the 9 hemispheres of the paired images with their test-retest reliability evaluated across the 2 normal, 3 MPTP, 2 implant, and 2 sham hemispheres.

\section{Regional Metabolic Effects of MPTP Administration and hRPE Cell Implantation}

Differences in regional glucose metabolism were assessed post hoc on a voxel basis with univariate SPM analysis. An unpaired or paired $t$ test was used to compare 7 MPTP and 8 normal scans or 6 hRPEimplanted and 6 MPTP scans, respectively. Analysis of covariance was used to account for interindividual variability in global metabolism. The differences were considered significant at a $P$ value of less than 0.005 with a cluster size of 20 voxels within a PRP-defined brain mask for hypothesis testing. The resulting maps of $t$ statistics were examined to localize relevant regions anatomically in reference to a macaque brain atlas.

\section{Statistical Analysis}

Unpaired $t$ tests were used to assess differences in network activity between animal groups. Paired $t$ tests were used to compare changes in motor ratings and in network scores between the MPTP and implant hemispheres or between the test and retest scans. The relationships between these measures and their interval changes were evaluated by correlation analysis. All analyses were performed using JMP software (version 5; SAS Institute) and were considered significant for a $P$ value less than 0.05 .

\section{RESULTS}

\section{Clinical Improvement by hRPE-GM Implantation}

At the preoperative baseline, motor ratings in the parkinsonian monkeys were $19.1 \pm 2.9$ (range 14.5-22.5) and comparable in the animals with hRPE-GM or sham implants (Fig. 1A). The hRPE-GM-implanted animals showed sustained motor improvement $(46.7 \% \pm 6.9 \%$ [mean $\pm \mathrm{SE}$ ]; range, $23 \%-66 \% ; P<0.001)$ compared with the preimplant scores and with the animals implanted with GM carriers only (Fig. 1B). The clinical improvement was robust in 4 animals (fetal cells) over 3-4 y and in 2 animals (neonatal cells) over 6-10 mon after hRPE-GM implantation. Motor ratings showed no changes in the 3 GM-implanted animals over 6-12 mon.

\section{Network Modulation by hRPE-GM Implantation}

Network activity changed in response to the implantation for PRPs 1-5 (Table 2; Fig. 3B; Supplemental Fig. 1B). In the 7 monkeys with unilateral implants, network scores were elevated in the untreated MPTP hemispheres relative to the normal values $(P<0.00005)$ but lower $(10.9 \%-26.8 \%, P<0.05 ; n=6)$ in the hRPE-GM-implanted versus the untreated MPTP hemispheres. Network scores in the hRPE-GM-implanted hemispheres were still higher than the normal values $(P<0.005)$. Network activity did not change in the 3 sham-operated animals (Supplemental Fig. 4). Treatment-mediated changes in network activity and clinical outcome did not correlate $\left(R^{2} \leq 0.18, P \geq 0.25\right)$ across the implant and sham-operated animals.

\section{Metabolic Differences Between Normal, Parkinsonian, and hRPE-GM-Implanted Macaques}

Hemispheric global metabolic values (in $\mathrm{kBq} / \mathrm{mL}$ ) did not change between the groups ( 8 normal, $46.0 \pm 6.4 ; 7$ MPTP, $54.6 \pm 3.5$ [mean $\pm \mathrm{SE}$ ]; $P=0.28$ ) or conditions (6 MPTP, $52.5 \pm 4.4 ; 6$ hRPE-implanted, $47.5 \pm 6.7 ; P=0.21)$. When the MPTP-lesioned were compared with the normal hemispheres, glucose metabolism increased in the putamen/pallidum, thalamus, pons, medial frontal gyrus/cingulate, sensorimotor cortex, and supplementary motor area but decreased in smaller areas in the posterior parietooccipital cortex (Fig. 4A; Table 3). Concurrently, glucose metabolism decreased in the same set of hypermetabolic regions but did not increase in the hRPEimplanted versus the MPTP-lesioned hemispheres (Fig. 4B; Table 3). 
TABLE 2

Changes in Metabolic Network Activity Under Different Experimental Conditions

\begin{tabular}{|c|c|c|c|c|c|}
\hline Summary of analysis & PRP1 & PRP2 & PRP3 & PRP4 & PRP5 \\
\hline \multicolumn{6}{|l|}{ Pattern derivation } \\
\hline Eigenvalue (\%) & 42.9 & 43.3 & 27.8 & 27.2 & 48.2 \\
\hline Control 1 & $0.00 \pm 0.41$ & $0.00 \pm 0.45$ & $0.00 \pm 0.36$ & $0.00 \pm 0.33$ & $0.00 \pm 0.41$ \\
\hline MPTP 1 & $7.67 \pm 1.37$ & $7.91 \pm 1.56$ & $3.11 \pm 0.25$ & $2.66 \pm 0.25$ & $7.78 \pm 1.41$ \\
\hline MPTP 1 vs. control $1^{\star}$ & 0.0037 & 0.0031 & 0.00003 & 0.00008 & 0.004 \\
\hline \multicolumn{6}{|l|}{ Pattern validation } \\
\hline Control 2 & $0.46 \pm 0.36$ & $0.09 \pm 0.36$ & $0.22 \pm 0.32$ & $0.36 \pm 0.28$ & $0.51 \pm 0.37$ \\
\hline MPTP 2 & $3.80 \pm 0.33$ & $3.88 \pm 0.32$ & $3.04 \pm 0.22$ & $2.64 \pm 0.21$ & $3.64 \pm 0.16$ \\
\hline MPTP 2 vs. control $2^{*}$ & 0.00001 & 0.000003 & 0.00001 & 0.00003 & 0.00002 \\
\hline \multicolumn{6}{|l|}{ Implant effect } \\
\hline Implant & $2.85 \pm 0.43$ & $3.10 \pm 0.48$ & $2.62 \pm 0.39$ & $1.92 \pm 0.35$ & $3.21 \pm 0.21$ \\
\hline Implant vs. MPTP 2 (change \%) & $-24.6 \pm 5.9$ & $-21.6 \pm 5.9$ & $-17.6 \pm 7.2$ & $-26.8 \pm 11.5$ & $-10.9 \pm 2.3$ \\
\hline Implant vs. MPTP $2^{\dagger}$ & 0.0025 & 0.011 & 0.036 & 0.033 & 0.0039 \\
\hline Implant vs. control $2^{*}$ & 0.001 & 0.0003 & 0.0005 & 0.004 & 0.00008 \\
\hline \multicolumn{6}{|l|}{ Test-retest effect } \\
\hline Test vs. retest ${ }^{\dagger}$ & 0.292 & 0.342 & 0.577 & 0.505 & 0.351 \\
\hline$R^{2}$ (Pearson correlation) & 0.954 & 0.952 & 0.953 & 0.956 & 0.926 \\
\hline$P$ & 0.000006 & 0.000007 & 0.000007 & 0.000005 & 0.00003 \\
\hline \multicolumn{6}{|c|}{$\begin{array}{l}{ }^{*} P \text { values: unpaired Student } t \text { tests. } \\
{ }^{\dagger} P \text { values: paired Student } t \text { tests. } \\
\text { Eigenvalue for each PRP derivation is given as percentage of total subject } \times \text { voxel variance (10). Subject scores are presented as mean } \pm \\
\text { E for animals used to identify each PRP and to assess effects of implantation. }\end{array}$} \\
\hline
\end{tabular}

\section{Test-Retest Reproducibility of Network Activity}

Network scores in the test-retest images remained unchanged $(P \geq 0.29)$ and were strongly correlated $\left(R^{2} \geq 0.926, P<\right.$ $0.00001)$ in the hemispheres of normal, MPTP, implanted, and sham-operated animals (Fig. 5; Supplemental Fig. 2). The results were similar across PRPs 1 to 5 .

\section{DISCUSSION}

The measurement of regional glucose metabolism with ${ }^{18} \mathrm{~F}$-FDG PET has been used extensively to assess the local and remote functional consequences of therapeutics in neurodegenerative disorders. This was shown by gene therapy in parkinsonian macaques (9) and cellular-based therapies in patients with multiple system atrophy (24) as well as in primate models of intracerebral hematoma (25) and rat models of cerebral ischemia (26). These studies documented widely distributed metabolic changes on a regional level but did not provide valuable information on a system level. We describe a complementary method of measuring both system and regional changes for evaluating treatment-mediated modulation of parkinsonism-related metabolic brain networks in primate models of PD.

In this study, we reported long-term effects of implanting hRPE-GM in MPTP-treated rhesus monkeys. Consistent motor recovery was present in the animals with unilateral hRPE-GM implants that remained stable over 36-48 mo but absent in those with GM only (Fig. 1B). This sustained improvement is similar to the predominantly contralateral improvements in off-state unified
Parkinson disease rating scale motor scores over 48 or 36 mo in PD patients implanted unilaterally with Spheramine (16) and hRPE-derived dopaminergic cells alone (27). Elevated PRP activity was significantly lower in the hRPE-GM-implanted than the untreated MPTP hemispheres (Fig. 3B) but did not change in the sham-operated hemispheres (Supplemental Fig. 4). This represented a robust effect of treatment given that PRP scores were elevated and highly symmetric in bilaterally lesioned animals without therapeutic intervention (10). These observations were consistently replicated for each of the 5 PRPs on a hemispheric or whole-brain basis.

We observed metabolic modulation in the same set of broadly distributed brain regions underlying the topography of PRP network. The regionally specific changes in glucose metabolism not only revealed downstream effects of MPTP lesioning and hRPEGM implants but also explained the elevated PRP activity in parkinsonian macaques and its subsequent suppression by this cell-based therapy. Of note, the changes in regional and network metabolic activity were independent of global metabolic values, which remained unchanged between animal groups or treatment conditions. The altered regional metabolism associated with the elevated PRP activity was similar in MPTP-lesioned cynomolgus monkeys scanned on a clinical PET/CT device (28).

Importantly, the changes in network and widespread regional metabolic activity after hRPE-GM implantation agree well with analogous observations in patients undergoing levodopa therapy and a variety of other neurosurgical interventions (5-7). These findings indicate that clinical response to symptomatic therapies 


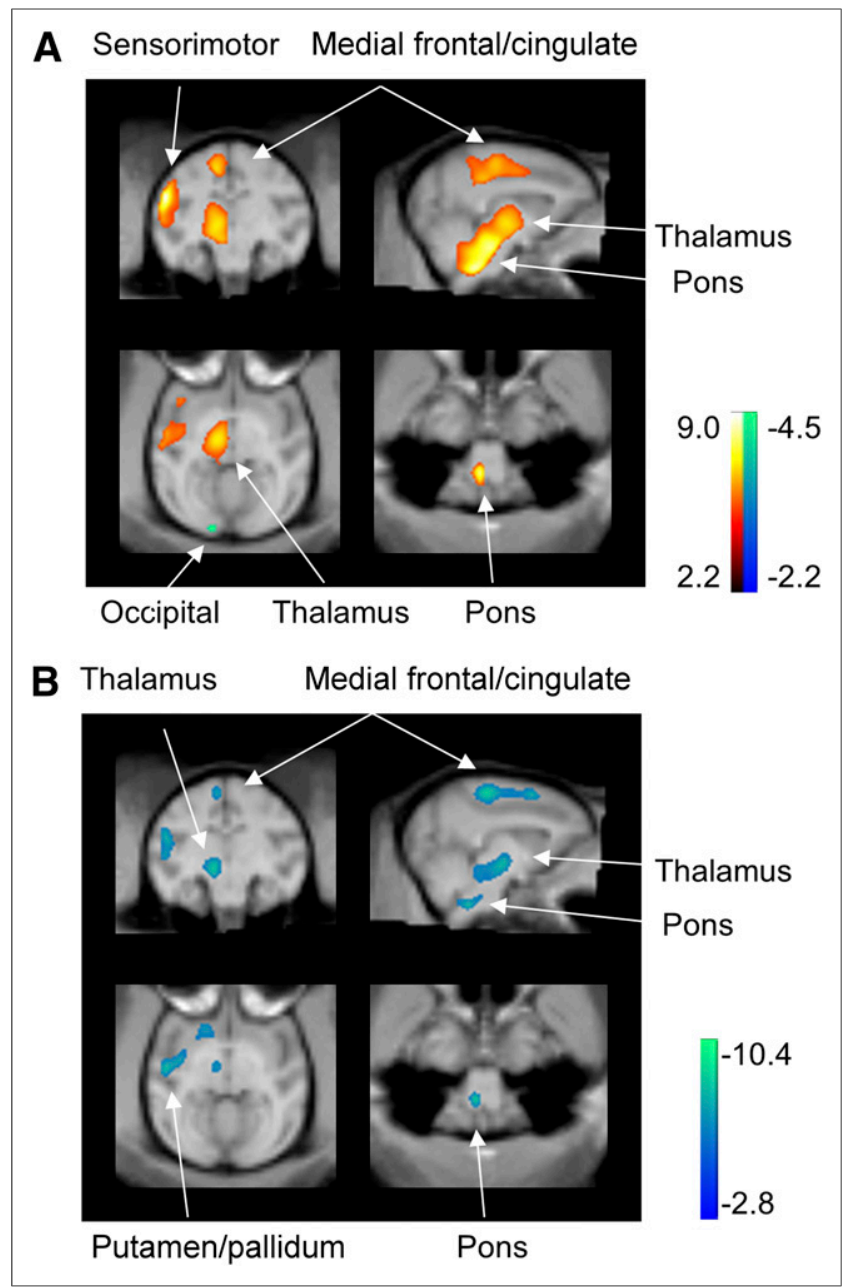

FIGURE 4. Modulation of abnormal regional metabolism in MPTPinduced experimental parkinsonism by hRPE cell transplantation therapy. (A) Metabolism in the 7 untreated MPTP hemispheres increased (red to yellow) in a set of subcortical and cortical motor regions relative to the 8 normal controls. (B) Metabolism in the $6 \mathrm{hRPE}$-implanted hemispheres decreased (blue to green) in the same set of subcortical and cortical motor regions compared with the 6 untreated MPTP hemispheres. SPM $t$ maps of unpaired and paired comparisons are displayed at lower threshold $(P=0.025)$ for better visualization on macaque MRI brain template (23).

is associated with the suppression of an elevated activity in PDrelated brain networks in both animal models and patients. We also showed high test-retest reliability of network activity (PRPs 1-5) in individual animals, regardless of clinical phenotype and treatment status, similar to the reports in human subjects $(1)$ and cynomolgus monkeys (28). Therefore, our cross-species studies have established that PD patients and primate models share homologous motor PD-related metabolic patterns that can be used prospectively for the assessment of disease severity and response to novel cell or gene therapies $(29,30)$.

In this study, we assumed implicitly that unilateral implantation of hRPE-GM cells causes metabolic effects mainly in the ipsilateral hemisphere because these nonneuronal cells do not appear to make synaptic connections with the host brain. Indeed, hRPErelated changes in dopaminergic markers were located only in the unilaterally implanted putamen in monkeys (14) and patients (27) along with contralateral clinical benefits. Many unilateral intervention studies make the same assumption so as to use the contralateral side as an internal control for the treatment side. The mean hemispheric difference in network activity was less than $6.5 \%$ in the 2 test-retest animals (2 and 3 ) who received the second implants in the contralateral striatum (supplemental data), much smaller than the effects of hRPE-GM implants (Table 2). Changes in metabolic value in remotely located regions and PDRP activity over the brain were also present only in the treated hemispheres plus improved contralateral motor ratings in PD patients after unilateral subthalamic gene therapy (6). All these results support the key assumption we made in this study.

The sham treatment involved only a small number of animals because of the ethical consideration of not performing invasive neurosurgery unnecessarily after we observed no clinical improvement in these animals. Our results are overall compatible with clinical findings in 6-hydroxydopamine-lesioned rats (12) and in the earlier masked sham-controlled study in hemiparkinsonian rhesus monkeys (13) that demonstrated significant clinical improvements in the animals implanted with hRPE-GM but not with sham (needle tracks) and microcarriers alone. These studies further suggest that control animals do not develop placebo responses as patients do.

We did not detect a significant association between the degree of network modulation and the clinical outcome in the limited sample of this study, because of the narrow range and variation of hRPE-mediated changes in both motor and network scores. Several sources of variability specific to our implantation therapy are described below. Despite this added variability, it is remarkable that each animal and each hemisphere in the hRPE-GMimplanted group demonstrates some level of reversal of parkinsonian attributes.

Our results revealed only a partial clinical recovery and suboptimal restoration of impaired brain circuitry by hRPE-GM implantation. This may be attributed to several factors: unilateral implantation, small numbers of implanted cells, lower rates of cell survival, poor integration with the host tissue, and properties of the cells themselves. It is important to remember that the hypothesized mechanism of action of striatally implanted hRPE cells is not the production of dopamine but constant in situ release of low levels of levodopa. The therapeutic effect of striatally implanted hRPE cells is thus indirect, through the increased stimulation of dopamine synthesis from surviving dopaminergic terminals, an action similar to that of orally administered levodopa but at much lower, physiologic concentrations. The incomplete functional recovery is consistent with clinical experience in patients with unilateral Spheramine implants $(15,22)$. These patients continued to receive daily levodopa treatment, albeit generally at much lower doses than before implant, to obtain optimal therapeutic benefit. Hence, neither Spheramine nor hRPE-GM cells at the concentrations given were sufficient to completely reverse the motor symptoms of parkinsonism.

It is fitting to provide some explanations for the discrepancy between our preclinical data and published clinical trial results. Motor symptoms of our monkeys were comparable to the patients in the phase I and II trials $(15,22)$ although their age ranges are closer to young patients in the phase I trial (15). Transplantation protocols were similar in both primates and patients with comparable numbers of tracts and cells implanted per side in the absence of immunosuppression. However, the hRPE cells used in this study were not all from the same donor and same origin (Table 1; Fig. 1). 
TABLE 3

Brain Regions with Significant Metabolic Changes Before and After hRPE Cell Implantation in Parkinsonian Macaques

\begin{tabular}{|c|c|c|c|c|c|c|c|c|c|c|}
\hline \multirow[b]{2}{*}{ Brain region } & \multicolumn{5}{|c|}{ Metabolic increase ${ }^{\star}$ (MPTP > normal) } & \multicolumn{5}{|c|}{ Metabolic decrease ${ }^{\dagger}$ (implant $<$ MPTP) } \\
\hline & $\mathrm{x}$ & $\mathrm{Y}$ & Z & $Z_{\max }$ & Size $\left(\mathrm{mm}^{3}\right)$ & $\mathrm{x}$ & $\mathrm{Y}$ & Z & $Z_{\max }$ & Size $\left(\mathrm{mm}^{3}\right)$ \\
\hline Medial frontal/cingulate & 8 & 6 & 28 & 3.3 & 376 & 4 & 6 & 36 & 2.9 & 208 \\
\hline Insula/SMC/putamen & 30 & 16 & 18 & 4.9 & 1304 & 26 & 8 & 18 & 3.0 & 744 \\
\hline Frontal/SMA & 28 & 24 & 18 & 4.5 & & 26 & 26 & 16 & 3.4 & 160 \\
\hline Thalamus & 6 & 14 & 8 & 2.9 & 1872 & 6 & 14 & 6 & 2.8 & 320 \\
\hline Pons & 4 & 0 & -14 & 3.9 & & 4 & 0 & -14 & 3.3 & 184 \\
\hline
\end{tabular}

*Unpaired $t$ test: $P<0.005$ uncorrected and survived at false-discovery rate-corrected $P<0.05$.

${ }^{\dagger}$ Paired $t$ test: $P<0.005$ uncorrected and did not survive at false-discovery rate-corrected $P<0.05$.

$\mathrm{SMC}=$ sensorimotor cortex; SMA = supplementary motor area.

Some were comparable to the fetal cells used in the successful open-label phase I trial (15) whereas others were similar to the neonatal cells used in the phase II trial that ultimately failed to demonstrate the efficacy of this intervention over placebo (22). Some of the variability in the behavioral and metabolic results may stem from the difference in cell sources. Indeed, several studies have shown that fetal hRPE cells have better survival characteristics than cells obtained from older donors (31). In our small group of animals, it was not possible to determine whether the overall behavioral improvement or PRP network recovery was driven mostly by the fetal cells' effects. Homogeneity of animals and similarity of MPTP disease model are also in sharp contrast with aging PD patients with multifactorial disease. One key difference in any double-blind, sham-controlled preclinical or clinical study is that animals do not develop placebo effects as compared

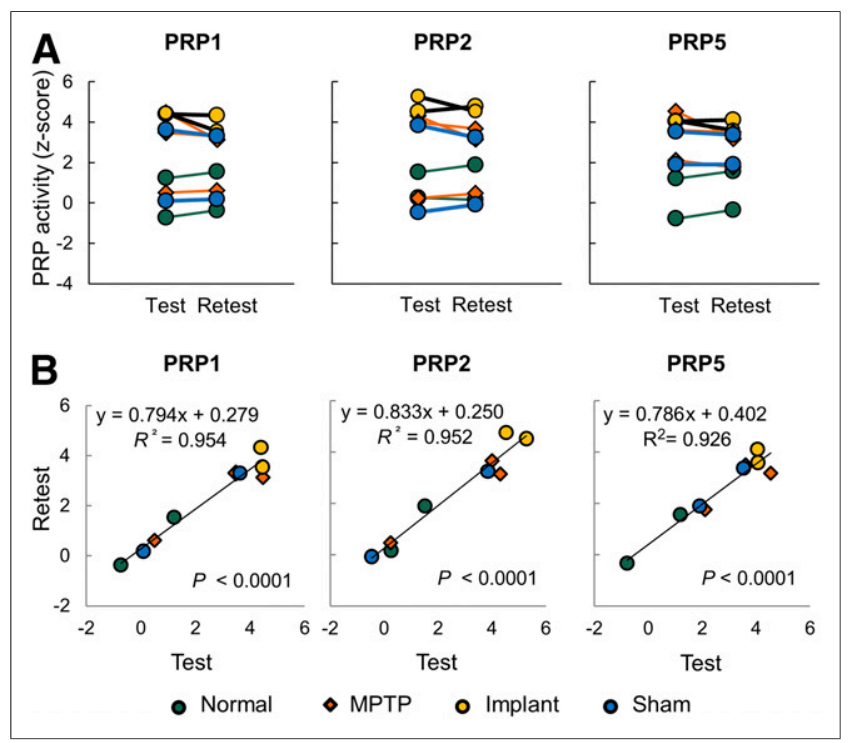

FIGURE 5. Test-retest reproducibility in PRP network activity. (A) Network scores in individual hemispheres or brains were highly reproducible (A: $P>0.29)$ and correlated (B: $\left.R^{2}>0.92 ; P<0.00005\right)$ between 9 test and retest scans in 4 subgroups of 7 macaques. Network scores were computed on hemispheric (PRPs 1-2) and whole-brain (PRP 5) basis, respectively. with patients undergoing the same trial. Nonetheless, the efficacy of this therapy may be improved by increasing the numbers of fetal-derived hRPE cells or enhancing their survival after implantation with the use of novel microcarriers (32).

There are several limitations in this study: small sample size, particularly in sham-operated control animals; unilateral implantation and subsequent analysis on a hemispheric basis; variable time intervals between implantation and imaging or between test and retest scans; and measurement of clinical motor scores rather than the more quantitative behavioral testing of the left versus right dexterity as in the monkey movement analysis panel. ${ }^{18}$ F-FDG PET was not part of the original study design and was added only after validation of the scanning procedure with glucose uptake in awake animals. Nevertheless, this study represents a simple proof of concept for the design of future preclinical trials. Within-subject designs with more potent or varied dose regimen will be necessary to delineate the effects of this or other potential therapies on metabolic network activity and correlations with behavioral outcome in a large sample before and after intervention.

\section{CONCLUSION}

We report, to our knowledge, the first study on the modulation of parkinsonism-related metabolic networks by experimental therapies in MPTP-treated primate models of PD. The covariance pattern of abnormal metabolism and network modulation in parkinsonian macaques parallel the reports in PD patients undergoing other symptomatic interventions. Changes in network activity may be useful for assessing the efficacy of novel therapeutics in both animal models and patients with PD. We also show a specific role of hRPE cells in the reversal of motor impairments in parkinsonian primates based on clinical benefits and suppression of network activity after implantation. These data support the use of ${ }^{18} \mathrm{~F}-\mathrm{FDG}$ PET to evaluate other formulations or microcarrier substrates with hRPEGM and other cell-based restorative therapies in patients with PD.

\section{DISCLOSURE}

The costs of publication of this article were defrayed in part by the payment of page charges. Therefore, and solely to indicate this fact, this article is hereby marked "advertisement" in accordance with 18 USC section 1734. This research was supported by Team Grant (CTP-79851) at the UBC from the Canadian Institute of 
Health Research. Dr. Doudet received grant-in-aid from Titan Pharmaceuticals and Bayer HealthCare Pharmaceuticals during this study but not for this study. Dr. Eidelberg serves on the scientific advisory board for and has received honoraria from the Michael J. Fox Foundation (MJFF) and The Bachmann-Strauss Dystonia and Parkinson Foundation; is listed as coinventor of patents regarding markers for use in screening patients for nervous system dysfunction and a method and apparatus for using same, without financial gain; has received research support from the NIH, High Q Foundation, the Dana Foundation, MJFF, and The Bachmann-Strauss Dystonia and Parkinson Foundation; has served as scientific advisor for the Thomas Hartman Foundation for Parkinson's Research, Inc.; and has received consultant fees from Pfizer Inc. Dr. Cornfeldt was employed by Titan Pharmaceuticals and owns stock purchased in the open market. Dr. Mitrovic was an employee of Bayer HealthCare Pharmaceuticals and is currently employed by Vertex Pharmaceuticals with stock ownership. Dr. Ma has received research support from the NIH. The work of Drs. Peng, Ma, and Eidelberg was supported by the NIH Morris K Udall Center of Excellence for Parkinson's Disease Research (P50 NS071675). No other potential conflict of interest relevant to this article was reported.

\section{ACKNOWLEDGMENTS}

We thank Titan Pharmaceuticals Inc. and Bayer HealthCare Pharmaceuticals for their gifts of the hRPE cells and microcarriers and the UBC/TRIUMF PET program for their assistance in imaging studies. Special thanks are due to the personnel of the UBC Animal Resources Unit and Animal Care Facilities for their outstanding care of the animals.

\section{REFERENCES}

1. Ma Y, Tang C, Spetsieris PG, Dhawan V, Eidelberg D. Abnormal metabolic network activity in Parkinson's disease: test-retest reproducibility. J Cereb Blood Flow Metab. 2007;27:597-605.

2. Wu P, Wang J, Peng S, et al. Metabolic brain network in the Chinese patients with Parkinson's disease based on ${ }^{18}$ F-FDG PET imaging. Parkinsonism Relat Disord. 2013;19:622-627.

3. Teune LK, Renken RJ, Mudali D, et al. Validation of parkinsonian disease-related metabolic brain patterns. Mov Disord. 2013;28:547-551.

4. Peng S, Ma Y, Spetsieris PG, et al. Characterization of disease-related covariance topographies with SSMPCA toolbox: effects of spatial normalization and PET scanners. Hum Brain Mapp. 2014;35:1801-1814.

5. Asanuma K, Tang C, Ma Y, et al. Network modulation in the treatment of Parkinson's disease. Brain. 2006;129:2667-2678.

6. Feigin A, Kaplitt MG, Tang C, et al. Modulation of metabolic brain networks after subthalamic gene therapy for Parkinson's disease. Proc Natl Acad Sci USA. 2007; 104:19559-19564.

7. Eidelberg D. Metabolic brain networks in neurodegenerative disorders: a functional imaging approach. Trends Neurosci. 2009;32:548-557.

8. Brownell AL, Canales K, Chen YI, et al. Mapping of brain function after MPTPinduced neurotoxicity in a primate Parkinson's disease model. Neuroimage. 2003;20:1064-1075.

9. Emborg ME, Carbon M, Holden JE, et al. Subthalamic glutamic acid decarboxylase gene therapy: changes in motor function and cortical metabolism. J Cereb Blood Flow Metab. 2007;27:501-509.

10. Ma Y, Peng S, Spetsieris PG, Sossi V, Eidelberg D, Doudet DJ. Abnormal metabolic brain networks in a nonhuman primate model of parkinsonism. J Cereb Blood Flow Metab. 2012;32:633-642.
11. Cepeda IL, Flores J, Cornfeldt ML, O'Kusky JR, Doudet DJ. Human retinal pigment epithelial cell implants ameliorate motor deficits in two rat models of Parkinson disease. J Neuropathol Exp Neurol. 2007;66:576-584.

12. Subramanian T, Marchionini D, Potter EM, Cornfeldt ML. Striatal xenotransplantation of human retinal pigment epithelial cells attached to microcarriers in hemiparkinsonian rats ameliorates behavioral deficits without provoking a host immune response. Cell Transplant. 2002;11:207-214.

13. Watts RL, Raiser CD, Stover NP, et al. Stereotaxic intrastriatal implantation of human retinal pigment epithelial (hRPE) cells attached to gelatin microcarriers: a potential new cell therapy for Parkinson's disease. J Neural Transm Suppl. 2003;65:215-227.

14. Doudet DJ, Cornfeldt ML, Honey CR, Schweikert AW, Allen RC. PET imaging of implanted human retinal pigment epithelial cells in the MPTP-induced primate model of Parkinson's disease. Exp Neurol. 2004;189:361-368.

15. Stover NP, Bakay RA, Subramanian T, et al. Intrastriatal implantation of human retinal pigment epithelial cells attached to microcarriers in advanced Parkinson disease. Arch Neurol. 2005;62:1833-1837.

16. Stover NP, Watts RL. Spheramine for treatment of Parkinson's disease. Neurotherapeutics. 2008;5:252-259.

17. Flores J, Cepeda IL, Cornfeldt ML, O’Kusky JR, Doudet DJ. Characterization and survival of long-term implants of human retinal pigment epithelial cells attached to gelatin microcarriers in a model of Parkinson disease. J Neuropathol Exp Neurol. 2007;66:585-596.

18. Farag ES, Vinters HV, Bronstein J. Pathologic findings in retinal pigment epithelial cell implantation for Parkinson disease. Neurology. 2009;73:10951102 .

19. Ma Y, Tang C, Chaly T, et al. Dopamine cell implantation in Parkinson's disease: long-term clinical and ${ }^{18}$ F-FDOPA PET outcomes. J Nucl Med. 2010;51:7-15.

20. Nandhagopal R, Kuramoto L, Schulzer M, et al. Longitudinal evolution of compensatory changes in striatal dopamine processing in Parkinson's disease. Brain. 2011;134:3290-3298.

21. Pavese N, Rivero-Bosch M, Lewis SJ, Whone AL, Brooks DJ. Progression of monoaminergic dysfunction in Parkinson's disease: a longitudinal ${ }^{18} \mathrm{~F}$-dopa PET study. Neuroimage. 2011;56:1463-1468.

22. Gross RE, Watts RL, Hauser RA, et al. Intrastriatal transplantation of microcarrierbound human retinal pigment epithelial cells versus sham surgery in patients with advanced Parkinson's disease: a double-blind, randomised, controlled trial. Lancet Neurol. 2011;10:509-519.

23. Black KJ, Koller JM, Snyder AZ, Perlmutter JS. Template images for nonhuman primate neuroimaging: 2. Macaque. Neuroimage. 2001;14:744-748.

24. Lee PH, Lee JE, Kim HS, et al. A randomized trial of mesenchymal stem cells in multiple system atrophy. Ann Neurol. 2012;72:32-40.

25. Feng M, Zhu H, Zhu Z, et al. Serial ${ }^{18}$ F-FDG PET demonstrates benefit of human mesenchymal stem cells in treatment of intracerebral hematoma: a translational study in a primate model. J Nucl Med. 2011;52:90-97.

26. Wang J, Chao F, Han F, et al. PET demonstrates functional recovery after transplantation of induced pluripotent stem cells in a rat model of cerebral ischemic injury. J Nucl Med. 2013;54:785-792.

27. Yin F, Tian ZM, Liu S, et al. Transplantation of human retinal pigment epithelium cells in the treatment for Parkinson disease. CNS Neurosci Ther. 2012;18: 1012-1020.

28. Ma Y, Johnston TH, Peng S, et al. Reproducibility of a Parkinsonism-related metabolic brain network in non-human primates: a descriptive pilot study with FDG PET. Mov Disord. 2015;30:1283-1288.

29. Ma Y, Peng S, Dhawan V, Eidelberg D. Dopamine cell transplantation in Parkinson's disease: challenge and perspective. Br Med Bull. 2011;100:173-189.

30. Bartus RT, Baumann TL, Brown L, Kruegel BR, Ostrove JM, Herzog CD. Advancing neurotrophic factors as treatments for age-related neurodegenerative diseases: developing and demonstrating "clinical proof-of-concept" for AAV-neurturin (CERE-120) in Parkinson's disease. Neurobiol Aging. 2013; 34:35-61.

31. Russ K, Flores J, Brudek T, Doudet D. Neonatal human retinal pigment epithelial cells secrete limited trophic factors in vitro and in vivo following striatal implantation in parkinsonian rats. J Neural Transm. 2016;123:167-177.

32. Falk T, Congrove NR, Zhang S, McCourt AD, Sherman SJ, McKay BS. PEDF and VEGF-A output from human retinal pigment epithelial cells grown on novel microcarriers. J Biomed Biotechnol. 2012;2012:278932. 